\title{
THE DEGREE OF PIECEWISE MONOTONE INTERPOLATION
}

\section{ELI PASSOW AND LOUIS RAYMON}

\begin{abstract}
Let $0=x_{0}<x_{1}<\cdots<x_{k}=1$ and let $y_{0}, y_{1}, \cdots, y_{k}$ be real numbers such that $y_{j-1} \neq y_{j}, j=1,2, \cdots, k$. Estimates are obtained on the degree of an algebraic polynomial $p(x)$ that interpolates the given data piecewise monotonely; i.e., such that (i) $p\left(x_{j}\right)=y_{j}, j=0,1, \cdots, k$, and such that (ii) $p(x)$ is increasing on $I_{j}=\left(x_{j-1}, x_{j}\right)$ if $y_{j}<y_{j-1}$, and decreasing on $I_{j}$ if $y_{j}<y_{j-1}, j=1,2, \cdots, k$. The problem is seen to be related to the problem of monotone approximation.
\end{abstract}

Let $0=x_{0}<x_{1}<\cdots<x_{k}=1$ and let $y_{0}, y_{1}, \cdots, y_{k}$ be real numbers such that $y_{j-1} \neq y_{j}, j=1,2, \cdots, k$. It is a result of Wolibner [7], Kammerer [2], and Young [8] that there exists an algebraic polynomial $p(x)$ such that:

(i) $p\left(x_{j}\right)=y_{j}, j=0,1, \cdots, k$, and

(ii) $p(x)$ is increasing on $I_{j}=\left(x_{j-1}, x_{j}\right)$ if $y_{j}>y_{j-1}$, and decreasing on $I_{j}$ if $y_{j}<y_{j-1}, j=1,2, \cdots, k$.

A polynomial $p(x)$ with properties (i) and (ii) is said to interpolate piecewise monotonely; in case $y_{j}>y_{j-1}$ for all $j$ (or if $y_{j}<y_{j-1}$ for all $j$ ), $p(x)$ is simply said to interpolate monotonely. The smallest degree of a polynomial that interpolates the values $Y=\left\{y_{0}, y_{1}, \cdots, y_{k}\right\}$ at the points $X=\left\{x_{0}, x_{1}, \cdots, x_{k}\right\}$ (piecewise) monotonely is called the degree of (piecewise) monotone interpolation of $Y$ with respect to $X$, and is denoted by $N=$ $N(X ; Y)$. Rubinstein has obtained estimates for the degree of monotone interpolation for the special case $k=2$ [6]. We seek general estimates on $N(X ; Y)$. Let

$$
\Delta=\Delta(Y)=\min _{1<j \leq k}\left|y_{j}-y_{j-1}\right|, \quad \text { and } \quad M=M(X ; Y)=\max _{1 \leq j \leq k}\left|\frac{y_{j}-y_{j-1}}{x_{j}-x_{j-1}}\right| \text {. }
$$

The estimates on $N(X ; Y)$ are found (not too surprisingly) to be related

Received by the editors January 30, 1974. $41 \mathrm{~A} 25$.

AMS (MOS) subject classifications (1970). Primary 41A05; Secondary 41A10,

Key words and phrases. Monotone interpolation, monotone approximation, comonotone approximation. 
to the degree of monotone and comonotone approximation. Let $f \in C[0,1]$, with a finite number of relative extrema $0=x_{0}<x_{1}<x_{2}<\cdots<x_{j+1}=1$ (such a function is called a piecewise monotone function). The relative extrema $x_{1}, x_{2}, \cdots, x_{j}$ are called the peaks of $f$. The degree of comonotone approximation of $f$ by algebraic polynomials of degree $\leq n$ is defined by

$$
E_{n}^{*}(f)=\inf \{\|f-p\|: p \in P(n ; f)\} \quad \text { (sup norm on }[0,1] \text { ), }
$$

where $P(n ; f)$ is the set of algebraic polynomials of degree $\leq n$, monotone on each of the subintervals $\left(x_{i-1}, x_{i}\right), i=1,2, \cdots, j+1$, with the same monotonicity as $f$ on these intervals. If $f$ is monotone on $[0,1]$, then $E_{n}^{*}(f)$ is called the degree of monotone approximation of $f$.

Let $S_{j}, j=0,1,2, \cdots$, be the set of all piecewise monotone functions $f$ with $j$ peaks such that

$$
\sup _{0 \leq x, y \leq 1}\left|\frac{f(x)-f(y)}{x-y}\right| \leq 1 .
$$

The degree of comonotone approximation to $S_{j}$ is defined by

$$
E_{n}^{*}\left(S_{j}\right)=\sup \left\{E_{n}^{*}(f): f \in S_{j}\right\}
$$

It is known that $\lim _{n \rightarrow \infty} E_{n}^{*}\left(S_{j}\right)=0$ [3], [4]. The smallest degree $n$ such that $E_{n}^{*}\left(S_{j}\right)<\delta$ will be denoted by $n_{j}(\delta)$.

With given data $X$ and $Y$ we associate a piecewise linear function $L(x)=$ $L(X ; Y ; x)$ defined by $L\left(x_{i}\right)=y_{i}, i=0,1, \cdots, k$. Let $j$ be the number of peaks of $L$. We state our main results:

Theorem 1. $N(X ; Y) \leq n_{j}(\Delta / 12 M)$.

Theorem 2. If $y_{0}<y_{1}<\cdots<y_{k}$, then there exists an absolute constant $A$ such that $N(X ; Y) \leq A M / \Delta$.

We will first prove Theorem 1. Theorem 2 will follow from Theorem 1 and estimates on the degree of monotone approximation. The proof is based on an idea of Kammerer [2, Theorem 4.1], later used by Ford and Roulier [1].

Proof of Theorem 1. Let $\epsilon=1 / 4 \Delta$, and let $S$ be the set of $2^{k+1}$ piecewise linear functions $f$ such that $f\left(x_{i}\right)=y_{i}+\epsilon$ or $y_{i}-\epsilon, i=0,1, \cdots, k$. We enumerate the functions in $S$ and denote the $i$ th function in $S$ by $f_{i}{ }^{\circ}$ Note that our choice of $\epsilon$ guarantees that each $f_{i}$ is comonotone with $L(x)$ and that $\left|f_{i}(x)-f_{i}(y)\right| \leq 3 M|x-y| / 2$ for all $x, y \in[0,1]$. Thus $2 f_{i} / 3 M \in S_{j}$, so that there exists $p_{i} \in P\left[n_{j}(\Delta / 12 M) ; L\right]$ such that $\left\|2 f_{i} / 3 M-p_{i}\right\|<\Delta / 12 M$. Let $q_{i}=$ $3 M p_{i} / 2$. Then $q_{i} \in P\left[n_{j}(\Delta / 12 M) ; L\right]$ and $\left\|f_{i}-q_{i}\right\|<(3 M / 2)(\Delta / 12 M)=\Delta / 8=\epsilon / 2$. 
The vector $\left(y_{0}, y_{1}, \cdots, y_{k}\right)$ is thus contained in the convex hull of the vectors $\left(q_{i}\left(x_{0}\right), q_{i}\left(x_{1}\right), \cdots, q_{i}\left(x_{k}\right)\right), i=1,2, \cdots, 2^{k+1}$, and therefore there exists a convex linear combination of the $q_{i}$ 's which will give rise to a polynomial $p$ which interpolates $Y$ piecewise monotonely. Since the degree of each $q_{i} \leq n_{j}(\Delta / 12 M)$, we have $N(X ; Y) \leq n_{j}(\Delta / 12 M)$.

Lemma (Lorentz and Zeller [3, Theorem 2]). There exists a constant $c$ such that $E_{n}^{*}\left(S_{0}\right) \leq c / n$.

Proof of Theorem 2. From the Lemma we obtain $n_{0}(\Delta / 12 M) \leq 12 c(M / \Delta)$. It follows from this and Theorem 1 that $N(X ; Y) \leq A M / \Delta$.

While Theorem 2 may fail to give the exact value of $N(X ; Y)$ for a particular configuration (e.g., if all the points lie on a straight line), it is best possible in a classwide sense, as we shall show in Theorem 3.

Let $G(M ; \Delta)$ be the set of all $(X ; Y)$ such that $M(X ; Y) \leq M$ and $\Delta(Y) \geq$ $\Delta$. Let $N(G)=\sup \{N(X ; Y):(X ; Y) \in G\}$.

Theorem 3. Let $y_{0}<y_{1}<\cdots<y_{k}$. Then there exist constants $c_{1}$, $c_{2}>0$ such that

$$
c_{1} M / \Delta \leq N(G) \leq c_{2} M / \Delta \text {. }
$$

Proof. For each $(X ; Y) \in G, N(X ; Y) \leq A M(X ; Y) / \Delta(Y)$, by Theorem 2 . Thus the upper bound in (2) holds with $c_{2}=A$.

For the lower bound, note that Theorem 2 was proved using estimates on $E_{n}^{*}\left(S_{0}\right)$ and that this theorem, in turn, may be used to give estimates on $E_{n}^{*}\left(S_{0}\right)$. Indeed, if $f \in C[0,1]$ is monotone and satisfies (1), we may choose $X=\left\{0=x_{0}, x_{1}, \cdots, x_{k}=1\right\}$ such that

$$
1 / n \leq f\left(x_{i}\right)-f\left(x_{i-1}\right) \leq 2 / n, \quad i=1,2, \ldots, k .
$$

Let $Y=\left\{f\left(x_{0}\right), f\left(x_{1}\right), \cdots, f\left(x_{k}\right)\right\}$. Then $\Delta(Y) \geq 1 / n$ and $M(X ; Y) \leq 1$ (by (1)), so that, by Theorem 2 , there exists a polynomial $p \in P\left(A_{n} ; f\right)$ such that $p\left(x_{i}\right)=f\left(x_{i}\right), i=0,1, \cdots, k$. Since $p$ is a monotone interpolation of $Y$ and $f$ satisfies (3), we have $\|f-p\| \leq 2 / n$. Thus $E_{A_{n}}^{*}(f) \leq 2 / n$, so that $E_{n}^{*}\left(S_{0}\right) \leq$ $A_{1} / n$, which is the result of Lorentz and Zeller contained in the Lemma. If $N(G)=o(M / \Delta)$, it would follow that there exists a sequence of polynomials $q_{n} \in P(n ; f)$ which would satisfy $\left\|f-q_{n}\right\|=o(1 / n)$. Since the result of Lorentz and Zeller is essentially unimprovable, this is impossible. Thus, there exists $c_{1}>0$ such that $N(G) \geq c_{1} M / \Delta$.

Remark. It follows from [4] and [5] that there exists $c_{3}>0$ such that 


$$
n_{j}(\Delta / 12 M) \leq \min _{P \geq j+2}\left[c_{3} P^{2} 2^{P} M / \Delta\right]^{(P+1) /(P-j-1)} .
$$

From this result we can obtain an estimate on $N(X ; Y)$ in the general case of $j$ peaks. We have, in particular, that for any $\epsilon>0$ there exists a constant $A_{j, \epsilon}$ such that

$$
N(X ; Y) \leq A_{j, \epsilon}(M / \Delta)^{1+\epsilon} .
$$

We believe, however, that this estimate is not the best possible one, since (4) is probably short of best possible. In fact, the proof of the lower bound (2) in Theorem 3 can be modified to show that in the general case of $j$ peaks, $N(G) \geq c_{4} M / \Delta$ for some constant $c_{4}$. We therefore conjecture that there exists $d_{j}$ such that $N(X ; Y) \leq d_{j} M / \Delta$, where $j$ is the number of peaks of $L(X ; Y ; x)$. It may even be possible to replace $d_{j}$ by an absolute constant $d$.

\section{REFERENCES}

1. W. T. Ford and J. A. Roulier, On interpolation and approximation by polynomials with monotone derivatives, J. Approximation Theory 10 (1974), 123-130.

2. W. J. Kammerer, Polynomial approximations to finitely oscillating functions, Math. Comp. 15 (1961), 115-119. MR 23 \#A1187.

3. G. G. Lorentz and K. L. Zeller, Degree of approximation by monotone polynomials. I, J. Approximation Theory 1 (1968), 501-504. MR 39 \#699.

4. E. Passow and L. Raymon, Monotone and comonotone approximation, Proc. Amer. Math. Soc. 42 (1974), 390-394.

5. E. Passow, L. Raymon and J. A. Roulier, Comonotone polynomial approximation, J. Approximation Theory 11 (1974), 221-224.

6. Z. Rubinstein, On polynomial $\delta$-type functions and approximation by monotonic polynomials, J. Approximation Theory 3 (1970), 1-6. MR 41 \#5844.

7. W. Wolibner, Sur un polynôme d'interpolation, Colloq. Math. 2 (1951), 136137. MR 13, 343 .

8. S. W. Young, Piecewise monotone polynomial interpolation, Bull. Amer. Math. Soc. 73 (1967), 642-643. MR 35 \#3326.

DE PAR TMENT OF MATHEMATICS, TEMPLE UNIVERSITY, PHILADELPHIA, PENNSYLVANIA 19122 